\title{
Arthroscopic treatment and mid-term results of suprapatellar plica syndrome: a single-center experience of 14 cases
}

\author{
Murat Aydın $\odot$, Selim Çınaroğlu²๑ \\ ${ }^{1}$ Department of Orthopedics and Traumatology, Niğde Ömer Halisdemir University School of Medicine, Niğde, Turkey \\ ${ }^{2}$ Department of Anatomy, Niğde Ömer Halisdemir University School of Medicine, Niğde, Turkey
}

\begin{abstract}
Objectives: Synovial plicae syndrome (SPS) are encountered as a mesenchymal tissue remnant during the process of embryological development, and divide the knee joint into three separate compartments. Of these, the suprapatellar plica may be affected by a syndrome that manifests with anterior knee pain, a clicking sound, snagging and a feeling of skipping after a patellofemoral overload. The present study evaluates 14 patients with suprapatellar complete plica syndrome who were treated between 2013 and 2019, and discusses the medical and surgical medium-term results of the condition.

Methods: Included in the study were 225 cases who underwent knee arthroscopy between 2013 and 2019, the data of which were analyzed retrospectively. The exclusion criteria were accompanying meniscal tear, anterior and posterior cruciate ligament tears, chronic inflammatory arthritis, varus $>3^{\circ}$ and valgus $>5^{\circ}$ malalignments. The cases were followed-up using the Tegner Lysholm knee scoring method, during the preoperative period, in the postoperative period, and in postoperative week 12 and month 6 . The statistical analysis was performed using the IBM SPSS Statistics (Windows Version 25.0. Armonk, NY: IBM Corp.) software package.

Results: A retrospective analysis revealed a BMI of $28.7 \pm 5.6 \mathrm{~kg} / \mathrm{m} 2$ in the 14 cases. The mean age was 34.57 \pm 8.53 years. Intraarticular cortisone injections and intraarticular viscosupplementations were performed at the outpatient follow-up in eight and three cases, respectively. Furthermore, eight cases had undergone previous physical therapy and rehabilitation. The mean duration of symptoms was $1.4 \pm 0.8$ months. The Tegner Lysholm knee scores were $69.53 \pm 6.15,88.23 \pm 3.00$, and $93.76 \pm 3.83$ in the preoperative period, week 12 and month 6 , respectively.
\end{abstract}

Conclusions: In conclusion, arthroscopy can be considered an efficacious and diagnostic method in knee plica syndrome. Patient satisfaction is high in the medium-term.

Keywords: Suprapatellar plica syndrome, knee joint, arthroscopy

Nuprapatellar syndrome (SPS) usually presents in $\checkmark$ the third decade of life, [1-2] with complaints of anterior knee pain, cracking noises, stumbling feelings and a popping sensation after patellofemoral overloading [2-5]. The prevalence of SPS varies in different populations, although the general prevalence world- wide is $10 \%[2,3,6,7]$. Suprapatellar syndrome is classified as suprapatellar, mediopatellar, infrapatellar and lateral, depending on the anatomical location (Fig. 1 ), while the most common type is medial plica $[2,3$, $6,7]$.

Plica, in its normal state, is a thin, flexible and

3ow to cite this article: Aydın M, Çınaroğlu S. Arthroscopic treatment and mid-term results of suprapatellar plica syndrome: a single-center experience of 14 cases. Eur Res J 2022;8(1):98-102. DOI: 10.18621/eurj.848551

Address for correspondence: Murat Aydın, MD., Niğde Ömer Halisdemir University School of Medicine, Department of Orthopedics and Traumatology, Niğde, Turkey.E-mail: opr.murataydin@gmail.com,Tel: +90 5324528559 


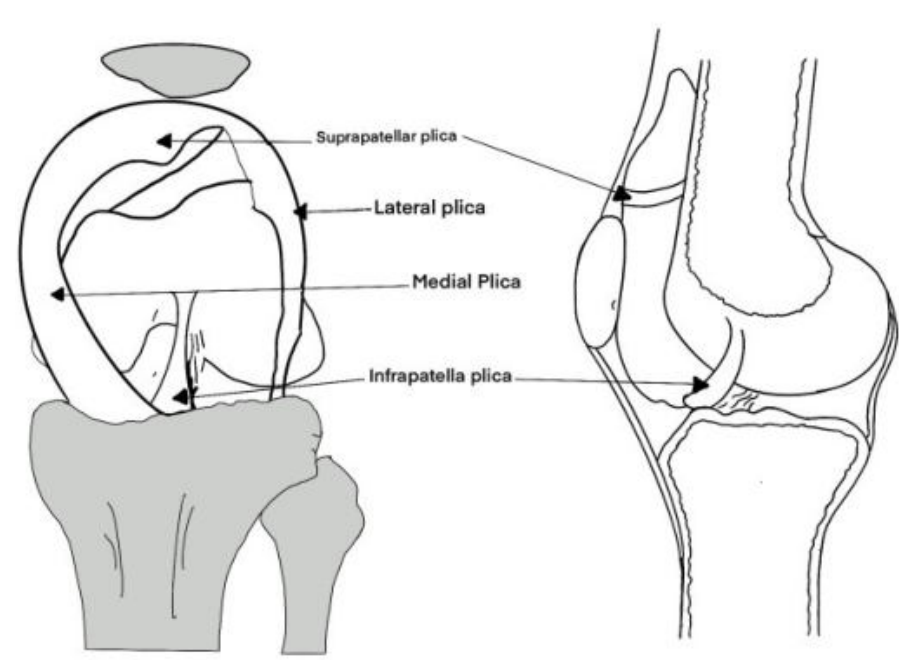

Fig. 1. Representative drawing of a suprapatellar, medial, infrapatellar and lateral plica of the knee.

transparent structure, and is associated with such mechanical complaints as pinching between the quadriceps tendon at the range of motion of the knee at 70-100 degree flexion, depending on the position of the plica.

Many SPS cases are idiopathic, and almost 60\% are bilateral [7]. Other causes are generally trauma, overuse, hematoma, diabetes mellitus and inflammatory arthropathy.

A total of 14 cases with suprapatellar complete plica syndrome who presented to our hospital between 2013 and 2019 were evaluated in the study, in which and medium-term medical and surgical results are dis- cussed.

The aim of this study is to discuss the success of arthroscopic surgery in suprapatellar plica syndrome. We believe that such a arthroscopic surgery will be a guide in the treatment of future patients, since patients with suprapatellar plica syndrome are mostly involved in terms of the characteristics of our hospital.

\section{METHODS}

Involved in the present study were 225 patients that underwent knee arthroscopy between 2013 and 2019, whose data were analyzed retrospectively. The exclusion criteria were accompanying meniscal tear, anterior and posterior cruciate ligament tears, chronic inflammatory arthritis, varus $>3^{\circ}$ and valgus $>5^{\circ}$ malalignments. The number of cases with no active pathological or anatomical findings other than plica syndrome and that underwent knee arthroscopy was 44 , according to the evaluations performed; 30 cases had a medial, lateral or infrapatellar plica; and a suprapatellar plica was present in 14 cases. Retrospective screening revealed a BMI of $28.7 \pm 5.6 \mathrm{~kg} / \mathrm{m} 2$ among the 14 cases included in the study, in which the mean age was $34.57 \pm 8.53$ years. Intraarticular cortisone injections and intraarticular viscosupplementations were performed at outpatient follow-up in eight and three cases, respectively. Furthermore, eight cases were found to have undergone physical therapy and reha-

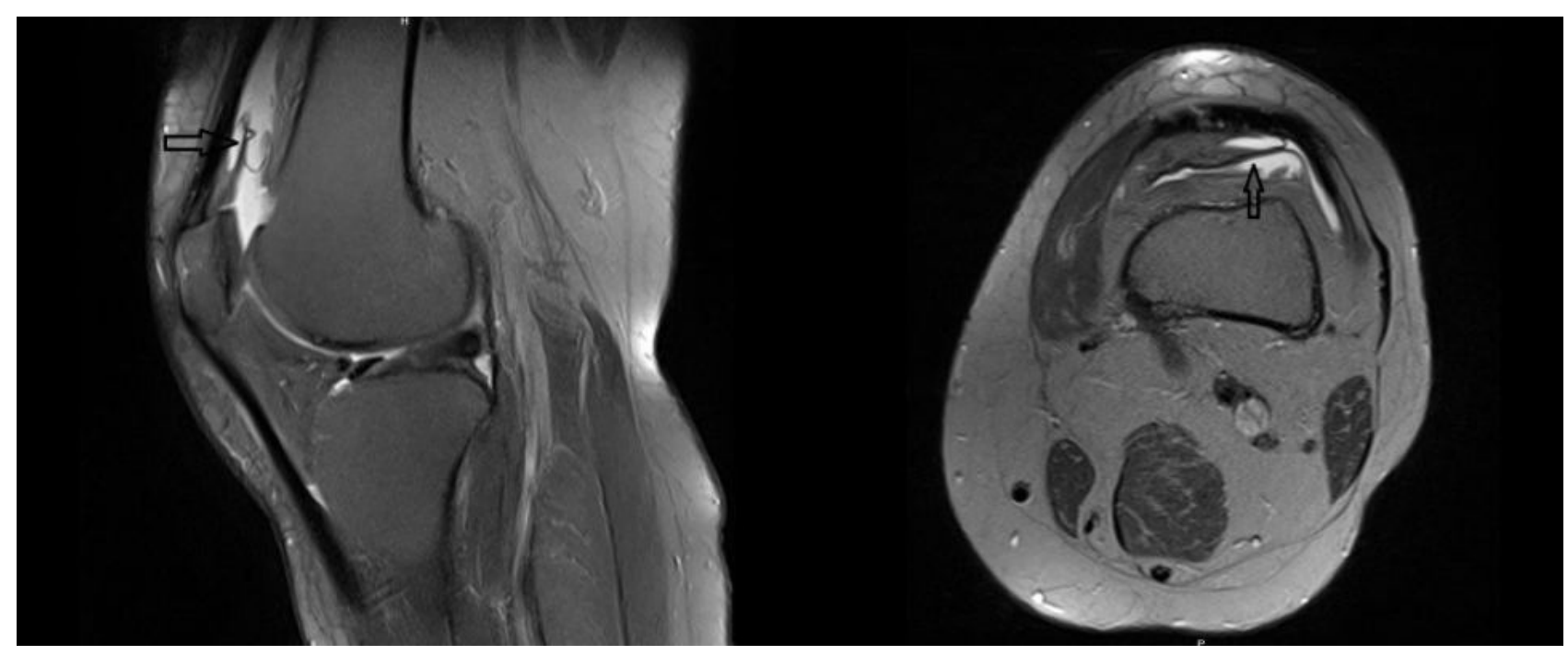

Fig. 2. Suprapatellar plica in the Sagittal and Transverse planes on MR measurement (black arrow). 
Table 1. Characteristic features of the cases.

\begin{tabular}{|c|c|c|c|c|c|c|c|c|}
\hline Cases & Age & $\begin{array}{l}\text { Intraarticular } \\
\text { cortisone } \\
\text { injection }\end{array}$ & $\begin{array}{c}\text { Intraarticular } \\
\text { Viscosupplem } \\
\text { entation }\end{array}$ & $\begin{array}{c}\text { Physical } \\
\text { therapy and } \\
\text { rehabilitation } \\
\text { previously }\end{array}$ & $\begin{array}{c}\text { Knee } \\
\text { pain }\end{array}$ & $\begin{array}{l}\text { Clicking } \\
\text { sound }\end{array}$ & Locking & Complications \\
\hline 1 & 32 & - & - & + & + & - & + & - \\
\hline 2 & 34 & - & - & + & + & - & + & - \\
\hline 3 & 36 & + & - & + & + & + & + & - \\
\hline 4 & 40 & + & - & + & - & - & + & - \\
\hline 5 & 23 & - & - & - & - & - & + & - \\
\hline 6 & 22 & - & - & - & - & + & + & - \\
\hline 7 & 43 & + & + & + & - & + & + & - \\
\hline 8 & 47 & + & + & + & + & - & + & + \\
\hline 9 & 45 & + & - & - & + & - & + & - \\
\hline 10 & 29 & - & - & - & - & + & - & - \\
\hline 11 & 35 & + & - & - & + & - & + & - \\
\hline 12 & 39 & + & + & + & + & - & + & - \\
\hline 13 & 39 & + & - & + & + & - & + & - \\
\hline 14 & 20 & - & - & - & - & + & + & - \\
\hline
\end{tabular}

bilitation previously. The mean duration of symptoms of the cases was $1.4 \pm 0.8$ months. The most frequent complaints were knee pain in eight cases, a clicking sound in six cases, a sense of stumbling or locking at over-flexion of the knee in 13 cases and a sense of popping after patellofemoral overload in 14 cases (Table 1). Plica syndrome was observed as an accompanying MRI finding in 10 cases (Fig. 2).

Written and verbal consent was obtained in all cases, and approval for the study was obtained from the Nigde Omer Halisdemir University, School of Medicine, Non-Interventional Clinical Research Ethics Board was taken.

\section{Surgical Technique}

The surgical field was entered via the standard antero-lateral port under spinal or general anesthesia and after a tourniquet application, scrubbing and draping. The antero-medial port was entered from the exterior to interior under direct vision. An arthroscopic exam-
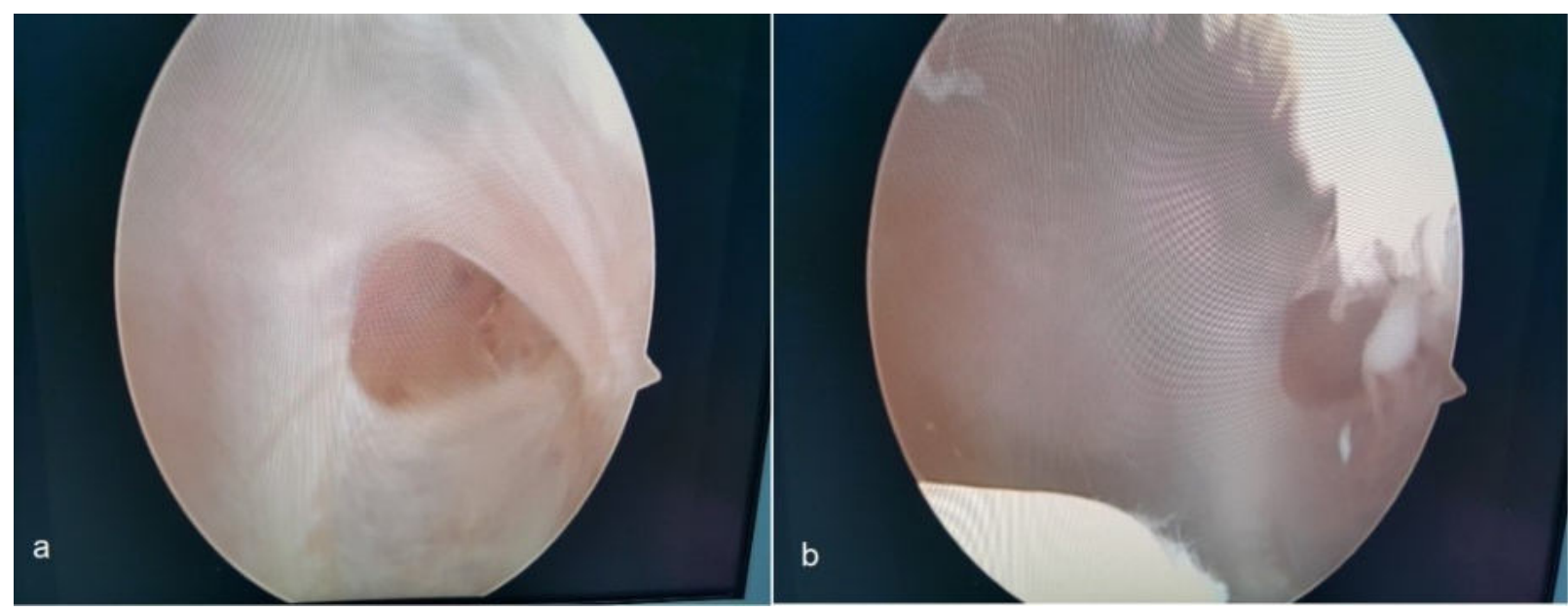

Fig. 3. (a) Suprapatellar plica during arthroscopy, (b) Suprapatellar space after debridement by arthroscopic tools. 


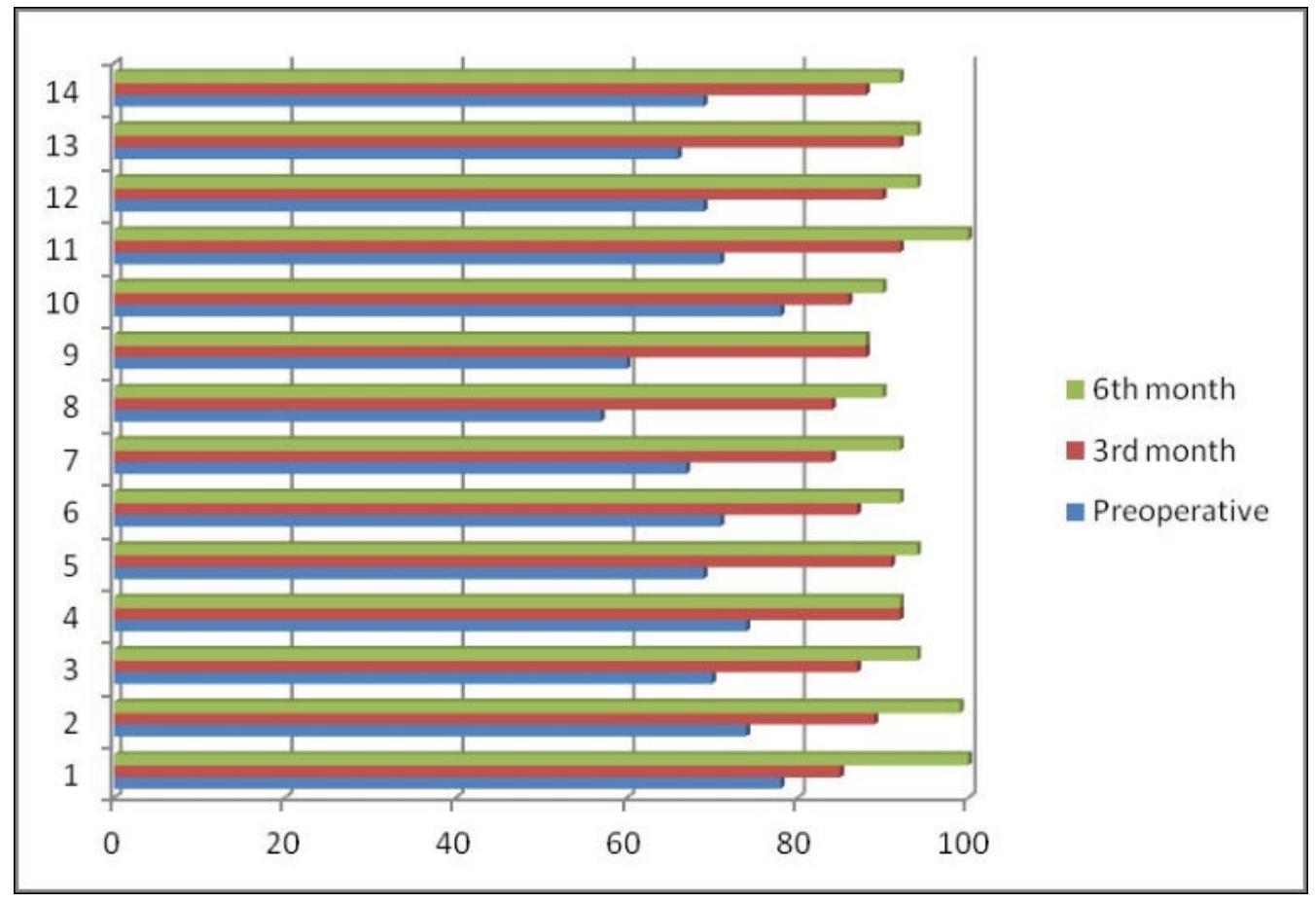

Fig. 4. Distribution of the cases according to the Tegner lysholm score preoperatively, at 3 and 6 months.

ination revealed an intact medial and lateral meniscus, anterior and posterior cruciate ligaments and cartilage tissues. A suprapatellar plica was observed and was released, and a debridement was performed (Fig. 3). A drain was placed and the skin was sutured. The wound was dressed and a Jones bandage was applied, and the sutures were removed on the postoperative 15 th day. A superficial skin infection developed on postoperative 6th day in one case and was treated with oral antibiotics. No serious complications developed in the remaining cases, other than the above.

\section{Statistical Analysis}

The cases were followed-up based on the Tegner Lysholm knee scoring method, applied during the preoperative period, postoperative period, and postoperative week 12 and month 6 [8]. Statistical analysis was performed using IBM SPSS Statistics (Windows Version 25.0. Armonk, NY: IBM Corp.).

\section{RESULTS}

The Tegner Lysholm knee scores of the cases were $69.53 \pm 6.15,88.23 \pm 3.00$ and $93.76 \pm 3.83$ in the preoperative period, at week 12 and month 6 , respec- tively. Significant differences were noted in the three control groups when the preoperative, week 12 and month 6 Tegner Lysholm knee scores were compared $(p<0.05)$ (Fig. 4).

\section{DISCUSSION}

Plica syndrome of the knee is a commonly problem of the knee in the adult population. The condition is frequently confused with osteochondritis dissecans, meniscal tears and degeneration, and patellar alignment disorders. Magnetic resonance imaging is a useful approach to differential diagnosis, although the sensitivity and specificity of axial MRI imaging, sagittal sections, and both sections when evaluated together are $73 \%$ and $78 \%, 71 \%$ and $83 \%$, and $95 \%$ and $72 \%$, respectively [9]. As such, it is necessary to consider additional pathologies in a differential diagnosis in cases involving plica syndrome.

Isolated plica cases may frequently be overlooked by clinicians during routine evaluations. A thickened plica can be palpated $1 \mathrm{~cm}$ medial to the upper pole of the patella in medial plica syndrome, and some patients might feel pain on palpation in the plica region as a result of this synovial thickening. Hughston's 
plica test and the Stutter test are provocative tests for plica syndrome, and may be applied concomitantly in physical examination [10-13].

The most reliable method in the treatment is arthroscopy, although intraarticular cortisone injections are also applied for the treatment of plica syndrome, being an efficacious diagnostic method and a highly detailed approach to treatment applied by clinicians $[1,2]$. A clear view of the lesion and intervention allows a $100 \%$ diagnosis, as was the case in this present study in which a significant difference was found in the Tegner-Lysom scores between the preoperative and postoperative periods, indicating clinical improvement. Tegner-Lysom is important to measure both function and activity level [8] and Also It has good criterion validity and test-retest reliability.

\section{Limitations}

The most significant limitation of the present study is its retrospective design, while further limitations include the small sample size and the fact that no MRIs were obtained postoperatively, as a follow-up MRI, after clinical improvement is considered unethical. The short duration of follow-up is another limitation of the study.

\section{CONCLUSION}

Arthroscopy can be considered an efficacious treatment and diagnostic approach in knee plica syndrome. Patient satisfaction is high in the short and medium term.

\section{Authors' Contribution}

Study Conception: MA, SÇ; Study Design: MA; Supervision: MA; Funding: MA, SÇ; Materials: MA; Data Collection and/or Processing: MA, SÇ; Statistical Analysis and/or Data Interpretation: MA; Literature Review: SÇ; Manuscript Preparation: MA, SÇ and Critical Review: MA, SÇ.

\section{Conflict of interest}

The authors disclosed no conflict of interest during the preparation or publication of this manuscript.

\section{Financing}

The authors disclosed that they did not receive any grant during conduction or writing of this study.

\section{REFERENCES}

1. Al-Hadithy N, Gikas P, Mahapatra AM, Dowd G. Review article: plica syndrome of the knee. J Orthop Surg (Hong Kong) 2011; 19(03):354-8.

2. Schindler OS. The Sneaky Plica' revisited: morphology, pathophysiology and treatment of synovial plicae of the knee. Knee Surg Sports Traumatol Arthrosc 2014;22:247-62.

3. Vassiou K, Vlychou M, Zibis A, Nikolopoulou A, Fezoulidis I, Arvanitis D. Synovial plicae of the knee joint: the role of advanced MRI. Postgrad Med J 2015;91:35-40.

4. Patel D. Plica as a cause of anterior knee pain. Orthop Clin North Am 1986;17:273-7.

5. Bellary SS, Lynch G, Housman B, Esmaeili E, Gielecki J, Tubbs RS, et al. Medial plica syndrome: a review of the literature. Clin Anat 2012;25:423-8.

6. Liu DS, Zhuang ZW, Lyu SR. Relationship between medial plica and medial femoral condyle--a three-dimensional dynamic finite element model. Clin Biomech (Bristol, Avon) 2013;28:1000-5.

7. Dupont JY. Synovial plicae of the knee. Controversies and review. Clin Sports Med 1997;16:87-122.

8. Tegner Y, Lysholm J. Rating systems in the evaluation of knee ligament injuries. Clin Orthop Relat Res 1985;198:43-9.

9. Jee WH, Choe BY, Kim JM, Song HH, Choi KH. The plica syndrome: diagnostic value of MRI with arthroscopic correlation.

J Comput Assist Tomogr 1998;22:814-8.

10. van der Heijden RA, Lankhorst NE, van Linschoten R, Bierma-Zeinstra SM, van Middelkoop M. Exercise for treating patellofemoral pain syndrome. Cochrane Database Syst Rev 2015; 1:CD010387.

11. McCunniff PT, Anthony CA, McDermott SE, Duchman KR, Albright JP. Arthroscopic validation of the clinical exam for the detection of the symptomatic synovial-plical complex by relief from surgical excision. Iowa Orthop J 2013;33:78-83.

12. Callaghan MJ, Selfe J. Patellar taping for patellofemoral pain syndrome in adults. Cochrane Database Syst Rev 2012;4:CD006717. 